\title{
Mineralização anaeróbia do nitrogênio em solos de várzea do Rio Grande do Sul
}

\author{
Nitrogen mineralization anaerobic in paddy soil from Rio Grande do Sul state, Brazil
}

\author{
Anderson Clayton Rhoden ${ }^{1}$ Leandro Souza da Silva ${ }^{2}$ Flávio Anastácio de Oliveira Camargo ${ }^{3}$ \\ Darines Britzke $^{4}$ Eliziane Luiza Benedetti ${ }^{4}$
}

\section{RESUMO}

$O$ potencial de mineralização do $N\left(N_{0}\right)$ do solo $e$ a taxa de mineralização $(k)$ são índices que podem ser usados na predição da disponibilidade do $\mathrm{N}$ em um determinado período de tempo que, juntamente com atributos de solo, podem estimar a absorção de $N$ por plantas de arroz irrigado. $O$ objetivo do presente trabalho foi de avaliar a mineralização do $N$ em 15 solos alagados do $R S$ e a sua relação com a absorção de $N$ por plantas de arroz irrigado. Utilizaram-se amostras de 15 solos da região arrozeira do RS, coletados de $0-20 \mathrm{~cm}$ de profundidade. Cultivaram-se plantas de arroz irrigado em vasos com quatro $\mathrm{kg}$ de cada solo durante 60 dias. A mineralização do $N$ foi avaliada em um experimento de incubação anaeróbia em laboratório, por 24 semanas, drenando-se a solução de alagamento dos solos semanalmente até a 14ํㅗ semana de incubação, a cada duas semanas até a $18^{a}$ semana de incubação e a cada três semanas até a 24ạa, totalizando 169 dias de incubação anaeróbia. O teor de C orgânico do solo está altamente associado ao $N$ absorvido pelas plantas $(r=0,90 ; P<0,01)$, bem como ao $N$ mineralizado $(r=0,91 ; P<0,01)$ e este ao teor de $N$ absorvido pelas plantas $(r=0,92 ; P<0,01)$. Os valores de $N_{0}$ para os quinze solos variaram de 107,7 a 207,6 $\mathrm{mg} \mathrm{kg}^{-1}$ e foram ligeiramente superiores ao $\mathrm{N}$ mineralizado observado $\left(98,3\right.$ a 191,3mg $\mathrm{kg}^{-1}$ de solo). A taxa de mineralização variou de 0,1492 a 0,2438 semana $^{-1}$ e a velocidade de mineralização foi maior no período inicial, compreendido até a quarta semana de incubação. Verificou-se uma alta associação entre o teor de $N$ absorvido por plantas de arroz irrigado e o $\mathrm{N}$ potencialmente mineralizável e deste com o teor de $C$ orgânico do solo. A taxa de mineralização foi relacionada significativamente com o teor de argila do solo $(r=0,77 ; P<0,01)$. O potencial de mineralização do $N$ pode ser utilizado como índice da disponibilidade de $N$ para a cultura do arroz irrigado.
Palavras-chave: arroz irrigado, $N$ absorvido, $N$ mineralizado, potencial de mineralização, taxa de mineralização.

\section{ABSTRACT}

The soil nitrogen mineralization potential $\left(N_{0}\right)$ and mineralization rate $(k)$ are indexes that can be used to predict $N$ availability in a period of time, which associated with soil attributes, can explain $N$ absorption in flooded rice plants. The objective of this study was to evaluate the $N$ mineralization in 15 flooded soils of Rio Grande do Sul (RS) state and its relationship with $N$ absorption by flooded rice plants. Samples collected from 0-0.2m layer of 15 soils from rice paddy fields in the RS state were used for the mineralization experiment. Flooded rice plants were grown for 60 days in pots containing four $\mathrm{kg}$ of each soil. The $N$ mineralization was evaluated in a laboratory experiment, in anaerobic conditions for 24 weeks. The content of soil organic $C$ was highly associated to the $N$ absorbed by the plants $(r=0.90 ; P<0.01)$, as well as to the mineralized $N(r=0.91 ; P<0.01)$ and to the content of $N$ absorbed by the plants $(r=0.92 ; P<0.01)$. The $N_{0}$ values for the fifteen soils varied from 107.70 to $207.64 \mathrm{mg} \mathrm{kg}^{-1}$ and they were higher for observed mineralized $N$ (98.3 to $191.3 \mathrm{mg} \mathrm{kg}^{-1}$ of soil). The mineralization rate constant varied from 0.1492 to 0.2438 week $^{-1}$, and the mineralization speed was higher at initial period, until the fourth week of incubation. It was verified a close relationship between the $N$ absorbed by rice plants and the nitrogen mineralization potential and with the content of soil organic $C$. The mineralization rate was significantly related to the clay content of the soil $(r=0.77 ; P<0.01)$. The potential of $N$ mineralization can be used as an index of $N$ availability to rice flooded plants.

Key words: flooded rice, absorbed $N$, mineralized $N$, nitrogen mineralization potential, and mineralization rate.

${ }^{1}$ Projeto PRAPEM/ Microbacias, Itapiranga, SC, Brasil. E-mail: andersonrhoden@mail.ufsm.br.

${ }^{2}$ Departamento de Solos, Centro de Ciências Rurais (CCR), Universidade Federal de Santa Maria (UFSM), 97105-900, Santa Maria, RS, Brasil. E-mail: leandro@smail.ufsm.br. Autor para correspondência.

${ }^{3}$ Departamento de Solos, Universidade Federal do Rio Grande do Sul (UFRGS), Porto Alegre, RS, Brasil. E-mail: faocamargo@bol.com.br.

${ }^{4}$ Curso de Agronomia, UFSM, Santa Maria, RS, Brasil. 


\section{INTRODUÇÃO}

A matéria orgânica do solo (MOS) é a principal fonte de carbono e de elétrons para os microrganismos do solo e fonte de nitrogênio $(\mathrm{N})$ para as plantas (PATRIK, 1982). Segundo CAMARGO et al. (1999), a matéria orgânica é a principal fonte de $\mathrm{N}$ para as culturas e cerca de $95 \%$ do $\mathrm{N}$ do solo encontra-se em formas orgânica reduzidas, não disponíveis para as plantas. É através da decomposição da MOS que o Né liberado sob a forma mineral em diferentes velocidades, dependendo da sua recalcitrância e resistência ao ataque microbiano (CAMARGO et al., 1997). Portanto, cada solo possui capacidade intrínseca de fornecer $\mathrm{N}$ às plantas a partir da decomposição da MOS, em quantidades e taxas diferentes, que dependem, fundamentalmente, do tipo de solo, da atividade microbiana e das condições ambientais.

$\mathrm{O}$ potencial de mineralização do $\mathrm{N}\left(\mathrm{N}_{0}\right)$ do solo e a taxa de mineralização do $\mathrm{N}$ (k) são índices que podem ser usados na predição da disponibilidade do $\mathrm{N}$ em um determinado período de tempo (STANFORD \& SMITH, 1972; CAMARGO et al., 1997; CAMARGO et al., 1999). O potencial de mineralização do $\mathrm{N}$ é definido como a fração do N orgânico do solo suscetível a mineralização e, a taxa de mineralização é a velocidade com que o N é mineralizado (CAMARGO et al., 1997). $\mathrm{O}$ conhecimento das taxas de mineralização permite avaliar o suprimento, os estágios de maior disponibilidade e a necessidade de adubação nitrogenada para as culturas (PÖTTKER \& TEDESCO, 1979), podendo-se, a partir da estimativa destas variáveis, ajustar a recomendação de adubação nitrogenada para as culturas.

A quantidade de $\mathrm{N}$ mineralizado é função do conteúdo e da taxa de mineralização do $\mathrm{N}$ orgânico, sabendo-se que existe uma relação direta entre o $\mathrm{N}$ mineralizado e o $\mathrm{N}$ absorvido pelas plantas (CAMARGO et al., 1997). Segundo LI et al. (2003), a predição da quantidade de $\mathrm{N}$ inorgânico liberado a partir da mineralização da MOS é essencial para o desenvolvimento de práticas que maximizem a eficiência no uso do $\mathrm{N}$ e minimizem impactos adversos ao meio ambiente, principalmente quando se considera a grande mobilidade e dinâmica do $\mathrm{N}$, tanto em ambientes de sequeiro, como em ambientes alagados. Existem diversos modelos matemáticos que visam determinar a fração do $\mathrm{N}$ orgânico potencialmente mineralizável e a taxa com que este $\mathrm{N}$ é mineralizado. O modelo proposto por STANFORD \& SMITH (1972) baseia-se em equação matemática que descreve uma cinética de primeira ordem, considerando somente um compartimento de $\mathrm{N}$ mineralizável. A equação que descreve o modelo é:
$\mathrm{N}_{\mathrm{m}}=\mathrm{N}_{\mathrm{o}}$ (1-exp $\left.\mathrm{ext}^{-\mathrm{t}}\right)$, onde $\mathrm{N}_{\mathrm{m}}$ é o $\mathrm{N}$ mineralizado acumulado em um período de tempo ( $\left.\mathrm{mg} \mathrm{N} \mathrm{kg}^{-1}\right), \mathrm{N}_{\mathrm{o}}$ é o potencial de mineralização do $\mathrm{N}\left(\mathrm{mg} \mathrm{N} \mathrm{kg}^{-1}\right)$, $\mathrm{k}^{\mathrm{o}}$ a constante de mineralização (semana ${ }^{-1}$ ) e té o tempo (semanas).

CAMARGO et al. (1997) comentam que os experimentos que estudam o potencial de mineralização do $\mathrm{N}$ do solo estão fundamentalmente atrelados ao ajuste matemático, utilizando-se deste para explicar a resposta da atividade microbiana e a absorção de $\mathrm{N}$ pelas plantas, e associando os parâmetros dos modelos que possam explicar a absorção de $\mathrm{N}$ pelas plantas. Portanto, o objetivo do presente trabalho foi o de avaliar o potencial de mineralização do $\mathrm{N}$ e a taxa de mineralização em 15 solos alagados do RS e a sua relação com a absorção de $\mathrm{N}$ pelas plantas de arroz irrigado.

\section{MATERIAL E MÉTODOS}

Amostras de quinze (15) solos de várzea (0$20 \mathrm{~cm}$ de profundidade) de diferentes regiões arrozeiras do Estado do RS foram coletadas e trazidas à UFSM, contemplando ampla variação de características químicas e físicas. As 15 amostras de solos foram coletadas aleatoriamente em 13 diferentes municípios: Camaquã, Dom Pedrito, Uruguaiana (duas amostras), Caçapava do Sul, Santo Antônio da Patrulha, Santa Maria, São Gabriel, Cachoeirinha, Restinga Seca, Cachoeira do Sul (duas amostras), Santa Vitória do Palmar, Rosário do Sul e Paraíso do Sul. A associação entre os locais de coleta dos solos com a classificação brasileira foi realizada de acordo com STRECK et al. (2002) e, tendo em vista a semelhança de algumas classes de solos, foi utilizada na discussão a denominação dos solos pelo município onde foram coletados. Após a caracterização química dos solos, aplicou-se calcário dolomítico (PRNT 75\%) para elevação e padronização do $\mathrm{pH}$ em valores próximos a 5,5, utilizando-se o índice SMP (CQFS, 2004). Os solos foram separados em sacos plásticos contendo $4 \mathrm{~kg}$, onde permaneceram fechados, incubando por um período de 35 dias, em casa de vegetação. Após este período determinou-se o $\mathrm{N}$ total conforme descrito em TEDESCO et al. (1995), argila conforme EMBRAPA (1977) e C orgânico conforme NELSON \& SOMMERS (1982) (Tabela 1).

a) Absorção de N por plantas de arroz

Os solos foram transferidos para vasos com capacidade para 8L e, posteriormente, semeou-se arroz pré-germinado, cv IRGA-417, deixando-se oito plântulas por vaso. Após, realizou-se o desbaste das plântulas, deixando-se seis plantas por vaso. Quatro dias após o 
Tabela 1 - Classificação, teores de carbono orgânico, nitrogênio total e argila dos solos utilizados para o cultivo

\begin{tabular}{|c|c|c|c|c|c|}
\hline Município & Unidade de Mapeamento & Classificação Brasileira & $\mathrm{C}$ org. & $\mathrm{N}$ total & Argila \\
\hline & & & \multicolumn{3}{|c|}{---------------g kg'1------------ } \\
\hline Camaquã & Itapeva & Gleissolo Melânico & 37,2 & 4,2 & 800 \\
\hline Dom Pedrito & Banhado & Gleissolo Háplico & 11,1 & 3,2 & 440 \\
\hline Uruguaiana 1 & Escobar & Vertissolo Ebânico & 30,9 & 3,9 & 370 \\
\hline Uruguaiana 2 & Uruguaiana & Chernossolo Ebânico & 20,0 & 2,6 & 330 \\
\hline Caçapava do Sul & São Gabriel & Planossolo Háplico & 16,2 & 2,0 & 320 \\
\hline$S^{o}$ Antônio da Patrulha & Vacacaí & Planossolo Hidromórfico & 11,8 & 1,9 & 250 \\
\hline Santa Maria & Vacacaí & Planossolo Hidromórfico & 9,5 & 1,8 & 240 \\
\hline São Gabriel & São Gabriel & Planossolo Háplico & 13,8 & 1,8 & 230 \\
\hline Cachoeirinha & Banhado & Gleissolo Háplico & 10,9 & 1,7 & 200 \\
\hline Restinga Seca & Vacacaí & Planossolo Hidromórfico & 8,3 & 1,0 & 190 \\
\hline Cachoeira do Sul 1 & Vacacaí & Planossolo Hidromórfico & 5,6 & 1,1 & 180 \\
\hline Cachoeira do Sul 2 & Vacacaí & Planossolo Hidromórfico & 4,4 & 0,9 & 130 \\
\hline Santa Vitória do Palmar & Pelotas & Planossolo Hidromórfico & 9,2 & 1,3 & 120 \\
\hline Rosário do Sul & São Gabriel & Planossolo Háplico & 5,5 & 0,8 & 90 \\
\hline Paraíso do Sul & Vacacaí & Planossolo Hidromórfico & 7,3 & 0,7 & 80 \\
\hline
\end{tabular}

transplante os solos foram alagados e, dez dias após a semeadura, elevou-se a lâmina de água a $5 \mathrm{~cm}$ de altura. Foram utilizadas quatro repetições por tratamento, num total de 60 vasos, dispostos em delineamento completamente casualizado. A adubação do arroz constou da adição de fósforo (P), aplicado em diferentes doses para cada solo a fim de manter na solução do solo uma concentração de $0,2 \mathrm{mg} \mathrm{L}^{-1}$, sendo esta uma concentração adequada de P para o desenvolvimento das culturas (NOVAIS \& SMITH, 1999), baseado na Capacidade Máxima de Adsorção de P de cada solo, conforme descrito em RANNO (2004). A fonte de P utilizada foi fosfato de potássio monobásico p.a. $\left(\mathrm{KH}_{2} \mathrm{PO}_{4}\right)$. Considerando-se o fato de que a fonte de $\mathrm{P}$ utilizada contém potássio $(\mathrm{K})$ e que a aplicação de diferentes doses de $\mathrm{P}$ implicou na adição de diferentes doses de K, foi realizado um complemento a adubação potássica utilizando-se $\mathrm{KCl}$ p.a. aos tratamentos que receberam menos $\mathrm{K}$, buscando-se obter a mesma adubação potássica para todos os vasos $\left(100 \mathrm{mg} \mathrm{kg}^{-1}\right.$ de $\left.\mathrm{K}_{2} \mathrm{O}\right)$. Aplicaram-se também os seguintes micronutrientes: ácido bórico $\left(4 \mathrm{mg} \mathrm{kg}^{-1}\right)$ sulfato de cobre $\left(3 \mathrm{mg} \mathrm{kg}^{-1}\right)$, cloreto de zinco $\left(1 \mathrm{mg} \mathrm{kg}^{-1}\right)$ e molibdato de sódio $\left(0,5 \mathrm{mg} \mathrm{kg}^{-1}\right)$. Sessenta dias após o transplante realizou-se a colheita da parte aérea das plantas, as quais foram levadas à estufa de ventilação forçada a $60^{\circ} \mathrm{C}$, onde permaneceram secando por cinco dias. Após, foram pesadas, moídas e determinado o teor de $\mathrm{N}$ nas plantas, conforme descrito em TEDESCO et al. (1995).

\section{b) Estimativa do potencial de mineralização}

Para o estudo do potencial de mineralização do N utilizou-se $25 \mathrm{~g}$ de cada solo calcareado misturado a igual quantidade de areia lavada, acomodado sobre
$25 \mathrm{~g}$ de areia lavada e cerca de $2 \mathrm{~cm}$ de altura de lã-devidro, acondicionados em tubos de lixiviação (100ml) em triplicatas. Os solos foram alagados com $30 \mathrm{ml} \mathrm{de}$ solução nutritiva contendo sulfato de magnésio, $\mathrm{MgSO}_{4}$ $\left(0,002 \mathrm{~mol} \mathrm{~L}^{-1}\right)$ e fosfato de cálcio monobásico, $\mathrm{Ca}\left(\mathrm{H}_{2} \mathrm{PO}_{4}\right)_{2}\left(0,005 \mathrm{~mol} \mathrm{~L}^{-1}\right)$, conforme POTTKËR \& TEDESCO (1979) e GONÇALVES et al. (2001). Os tubos foram cobertos com filme plástico contendo um orifício central e incubados em estufa a $30^{\circ} \mathrm{C}$. A solução de alagamento dos solos foi drenada semanalmente até a 14ª semana de incubação, a cada duas semanas até a $18^{\mathrm{a}}$ semana de incubação e a cada três semanas até a 24a , totalizando 169 dias de incubação anaeróbia, com o auxílio de uma bomba de vácuo e pesada para que se retornasse os tubos um volume próximo aos $30 \mathrm{ml}$ iniciais com adição de água destilada. $\mathrm{O}$ procedimento detalhado para tal encontra-se descrito em RHODEN (2005). Na solução drenada foi determinado o teor de $\mathrm{N}-\mathrm{NH}_{4}{ }^{+}$e os teores de $\mathrm{N}-\left(\mathrm{NO}_{3}{ }^{-}+\mathrm{NO}_{2}{ }^{-}\right)$conforme TEDESCO et al. (1995).

O potencial de mineralização do $\mathrm{N}\left(\mathrm{N}_{0}\right)$ e as taxas de mineralização (k) foram obtidos a partir dos valores de $\mathrm{N}$ mineralizado acumulado $\left(\mathrm{N}_{\mathrm{m}}\right)$ em função do tempo, utilizando-se o modelo matemático de STANFORD \& SMITH (1972): $\mathrm{N}_{\mathrm{m}}=\mathrm{N}_{\mathrm{o}}\left(1-\mathrm{exp}^{\mathrm{kt}}\right)$, onde $\mathrm{N}_{\mathrm{m}}$ é o $\mathrm{N}$ mineralizado acumulado em um período de tempo $\left(\mathrm{mg} \mathrm{N} \mathrm{kg}^{-1}\right), \mathrm{N}_{\mathrm{o}}$ é o potencial de mineralização do $\mathrm{N}\left(\mathrm{mg} \mathrm{N} \mathrm{kg}^{-1}\right)$, ké a constante de mineralização (semana ${ }^{-1}$ ) e t é o tempo (semanas). Após análise de variância, utilizaram-se os testes de Tukey, Duncan e teste-t $(\mathrm{P}<0,05)$ para comparar os valores médios de $\mathrm{N}$ mineralizado dos solos, formando-se grupos de acordo com as diferenças mínimas significativas indicadas 
pelos testes. Também foram realizadas correlações de Pearson entre algumas propriedades dos solos e o teor de $\mathrm{N}$ absorvido pelas plantas de arroz irrigado.

\section{RESULTADOS E DISCUSSÃO}

Os solos apresentaram diferentes quantidades de $\mathrm{N}$ mineralizado acumulado [soma de $\left.\mathrm{NH}_{4}+\left(\mathrm{NO}_{3}+\mathrm{NO}_{2}\right)\right]$ em condições anaeróbias num período de 24 semanas, variando de 98,3 a $191,3 \mathrm{mg} \mathrm{kg}^{-1}$ de solo (Tabela 2). O comportamento da mineralização do $\mathrm{N}$ acumulado com o tempo foi distinto entre os solos (Figura 1), o que permitiu separar, através das diferenças mínimas significativas dos testes de comparação de médias, grupos de solos com comportamento similar, assim como realizado por CAMARGO et al. (1997) ao estudar dez solos do RS em incubação aeróbia.

Ao se utilizar como referência para o agrupamento dos solos a diferença mínima significativa do teste de Tukey $(\mathrm{P}<0,05)$, formou-se dois grupos e, desta forma, 12 solos foram enquadrados no primeiro, apresentando uma média de $121,7 \mathrm{mg}$ de $\mathrm{N}$ mineralizado $\mathrm{kg}^{-1}$ de solo e os demais três solos restantes foram agrupados em valores de $\mathrm{N}$ mineralizado mais elevado (média de $180,9 \mathrm{mg} \mathrm{de}^{\mathrm{N} \mathrm{kg}}{ }^{-1}$ de solo) formado por Uruguaiana 2, Uruguaiana 1 e Camaquã. Já o uso de testes estatísticos menos rigorosos proporcionou a formação de um maior número de grupos, como o teste- t e o Duncan $(\mathrm{P}<0,05)$, que indicam a formação de cinco grupos de solos. O primeiro grupo é formado pelos seis solos com os menores valores de $\mathrm{N}$ mineralizado acumulado, numa média de $107,6 \mathrm{mg} \mathrm{kg}^{-1}$ de solo (Restinga Seca, Paraíso do Sul, Rosário do Sul, Cachoeira do Sul 1, Dom Pedrito e Cachoeira do Sul 2), o segundo grupo é formado por 5 solos com uma média de $132,5 \mathrm{mg} \mathrm{kg}^{-1}$ de solo de $\mathrm{N}$ mineralizado (Cachoeirinha, São Gabriel, Santa Vitória do Palmar, Santa Maria e Santo Antônio da Patrulha), o terceiro grupo é formado somente pelo solo de Caçapava, com $\mathrm{N}$ mineralizado de $151,7 \mathrm{mg} \mathrm{kg}^{-1}$ de solo, o quarto grupo é formado pelos dois solos de Uruguaiana, que apresentaram um $\mathrm{N}$ mineralizado médio de $175,6 \mathrm{mg} \mathrm{kg}^{-1}$ de solo e, o quinto grupo é formado somente pelo solo de Camaquã, com um $\mathrm{N}$ mineralizado de $191,3 \mathrm{mg} \mathrm{kg}^{-1}$ de solo.

Numa análise global, considerando a formação de cinco grupos de solos, observa-se que o primeiro grupo é formado por solos que possuem baixo teor de C orgânico e de $\mathrm{N}$ total e que são mais arenosos (Tabela 1). Estes componentes do solo poderiam explicar a baixa quantidade de $\mathrm{N}$ mineralizado acumulado final, com exceção para o solo de Dom Pedrito. O segundo grupo de solos possui maiores teores de $\mathrm{C}$ orgânico, de $\mathrm{N}$ total e de argila, o que poderia explicar a maior mineralização de $\mathrm{N}$ com relação ao primeiro grupo de solos, assim como os demais solos

Tabela 2 - Quantidade de $\mathrm{N}$ mineralizado final $(\mathrm{Nm}), \mathrm{N}$ potencialmente mineralizável $\left(\mathrm{N}_{0}\right)$, coeficiente de correlação, relação $\mathrm{N}_{0} / \mathrm{Nt}$, taxa de mineralização (k) e $\mathrm{N}$ absorvido por plantas de arroz irrigado após 60 dias de cultivo

\begin{tabular}{|c|c|c|c|c|c|c|}
\hline Município & $\mathrm{Nm}^{1}$ & $\mathrm{~N}_{0}{ }^{1}$ & $r^{2}$ & Relação $\mathrm{N}_{0} / \mathrm{Nt}$ & $\mathrm{k}^{1}$ & $\begin{array}{c}\mathrm{N} \text { absorvido } \\
\text { arroz }\end{array}$ \\
\hline & \multicolumn{2}{|c|}{-----mg kg-1----- } & & $\%$ & semana $^{-1}$ & $\mathrm{mg}$ vaso $^{-1}$ \\
\hline Camaquã $^{2}$ & 191,3 & 207,6 & 0,981 & 4,9 & 0,1492 & 257,7 \\
\hline Dom Pedrito $^{3}$ & 113,1 & 117,3 & 0,990 & 3,7 & 0,2036 & 88,1 \\
\hline Uruguaiana $1^{4}$ & 177,7 & 183,5 & 0,994 & 4,7 & 0,1984 & 307,7 \\
\hline Uruguaiana $2^{5}$ & 173,5 & 181,6 & 0,989 & 7,1 & 0,1836 & 278,6 \\
\hline Caçapava do $\mathrm{Sul}^{6}$ & 151,7 & 161,0 & 0,974 & 8,0 & 0,1881 & 130,1 \\
\hline $\mathrm{S}^{\circ}$ Antônio da Patrulha ${ }^{7}$ & 140,5 & 147,6 & 0,979 & 7,7 & 0,1935 & 104,4 \\
\hline Santa Maria $^{7}$ & 134,7 & 138,4 & 0,988 & 7,5 & 0,2379 & 91,7 \\
\hline São Gabriel $^{6}$ & 128,8 & 132,6 & 0,989 & 7,4 & 0,2292 & 114,7 \\
\hline Cachoeirinha $^{3}$ & 125,4 & 130,5 & 0,977 & 7,8 & 0,2120 & 71,7 \\
\hline Restinga Seca ${ }^{7}$ & 98,3 & 104,7 & 0,984 & 9,7 & 0,2313 & 24,7 \\
\hline Cachoeira do Sul $1^{7}$ & 122,3 & 117,3 & 0,975 & 10,3 & 0,2096 & 37,4 \\
\hline Cachoeira do Sul $2^{7}$ & 115,1 & 120,6 & 0,979 & 13,8 & 0,2132 & 44,8 \\
\hline Santa Vitória do Palmar ${ }^{8}$ & 133,1 & 137,1 & 0,989 & 10,8 & 0,2438 & 99,8 \\
\hline Rosário do $\mathrm{Sul}^{6}$ & 106,7 & 112,4 & 0,964 & 14,8 & 0,2097 & 57,4 \\
\hline Paraíso do $\mathrm{Sul}^{7}$ & 100,1 & 105,4 & 0,964 & 15,5 & 0,2133 & 42,8 \\
\hline
\end{tabular}

${ }^{1}$ Estimado pelo modelo de STANFORD \& SMITH (1972): $\mathrm{N}_{\mathrm{m}}=\mathrm{N}_{\mathrm{o}}\left(1-\mathrm{exp}^{\mathrm{kt}}\right)$

Unidade de Mapeamento e Classificação Brasileira $={ }^{2}$ Itapeva - Gleissolo Melânico; ${ }^{3}$ Banhado - Gleissolo Háplico; ${ }^{4}$ Escobar - Vertissolo Ebânico; ${ }^{5}$ Uruguaiana - Chernossolo Ebânico; ${ }^{6}$ São Gabriel - Planossolo Háplico; ${ }^{7}$ Vacacaí - Planossolo Hidromórfico; ${ }^{8}$ Pelotas - Planossolo Hidromórfico. 


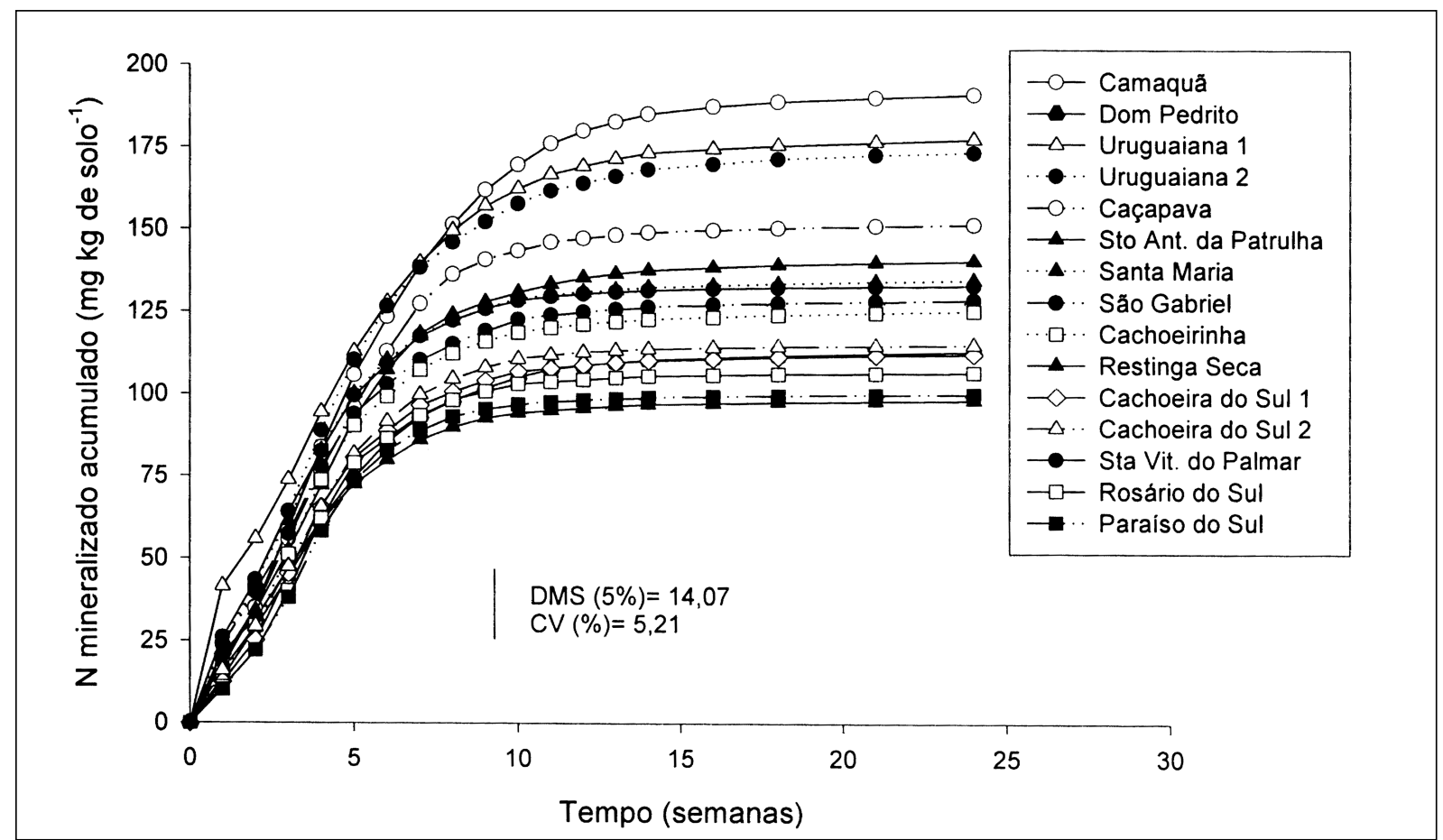

Figura 1 - Teor de $\mathrm{N}$ mineralizado acumulado em função do tempo de incubação anaeróbia. DMS = diferença mínima significativa pelo teste de Tukey a $5 \%$ de probabilidade; e Ç= coeficiente de variação experimental.

no terceiro e quarto grupos. Na figura 1 também se observa que a velocidade de mineralização do $\mathrm{N}$ foi maior no período inicial compreendido até a quarta semana, em relação aos períodos posteriores. Em média, $52 \%$ (39-58\%) do $\mathrm{N}$ mineralizado, foi obtido até a quarta semana de incubação. Estas constatações podem estar atreladas a decomposição da MOS mais lábil, sendo que, no geral, solos com maior teor de C orgânico e de $\mathrm{N}$ total apresentaram os maiores valores de $\mathrm{N}$ mineralizado acumulado. Entretanto, a partir da quinta semana de incubação os solos apresentaram maior diferença com relação ao $\mathrm{N}$ mineralizado acumulado, demonstrando as características intrínsecas de cada solo e a sua capacidade em mineralizar $\mathrm{N}$.

No período compreendido entre o início e a oitava semana de incubação e décima segunda, em média, o $\mathrm{N}$ mineralizado chegou a $80 \%$ (69-84\%) e $86 \%$ (82-88\%) do $\mathrm{N}$ mineralizado ao final de 24 semanas, respectivamente. A partir da oitava semana de incubação, o processo de mineralização aproximouse de uma cinética de ordem zero, com diminuição do $\mathrm{N}$ mineralizado em função do tempo, assim como o observado por CAMARGO et al. (1997). Nos solos do RS estudados por PÖTTKER \& TEDESCO (1979), as taxas de mineralização do $\mathrm{N}$ diminuíram sensivelmente após as três semanas iniciais de incubação, e concluíram que durante a incubação há uma rápida liberação inicial de $\mathrm{N}$, seguida de uma diminuição gradativa.

A mineralização do $\mathrm{N}$ depende de vários fatores, tais como: tipo de solo, conteúdo de matéria orgânica, relação $\mathrm{C} / \mathrm{N}$, tempo de uso dos solos, $\mathrm{pH}$, temperatura, umidade, secamento, congelamento, suprimento de nutrientes inorgânicos e as interações solo-planta (BLACK, 1968; PÖTTKER \& TEDESCO, 1979). De acordo com VAHL (1999), a taxa de liberação do $\mathrm{N}$ em solo que sofre alagamento também é dependente do tempo de alagamento e de eventuais ciclos de umedecimento e secagem. Entretanto, como os solos foram incubados a uma temperatura de $30^{\circ} \mathrm{Ce}$ sob condições de alagamento, a mineralização do $\mathrm{N}$ foi exclusivamente dependente das características intrínsecas de cada solo. $\mathrm{O} \mathrm{N}$ mineralizado acumulado em 24 semanas apresentou elevada e significativa associação com o $\mathrm{N}$ absorvido por plantas de arroz irrigado $(\mathrm{r}=0,92 ; \mathrm{P}<0,01 ; \mathrm{n}=15)$. Observa-se também que o teor de $\mathrm{C}$ orgânico está alto e significativamente correlacionado com o $\mathrm{N}$ mineralizado $(\mathrm{r}=0,91 ; \mathrm{P}<0,01$; $\mathrm{n}=15$ ), evidenciando a importância deste parâmetro na capacidade do solo em mineralizar $\mathrm{N}$ e na estimativa do $\mathrm{N}$ absorvido por plantas de arroz irrigado. De modo geral, o baixo conteúdo de $\mathrm{N}$ mineralizado pelos solos de Paraíso do Sul, Rosário do Sul, Cachoeira do Sul e Restinga Seca pode ser atribuído aos baixos teores de 
C orgânico e de $\mathrm{N}$ total destes solos, efeito contrário pode ser observado com os solos que apresentaram elevados teores de $\mathrm{N}$ mineralizado, com exceção do solo Dom Pedrito (Tabela 1).

Quando se avalia a correlação N mineralizado e $\mathrm{N}$ total do solo, obtém-se uma associação significativa $(\mathrm{r}=0,80 ; \mathrm{P}<0,01 ; \mathrm{n}=15)$. Diversos são os trabalhos que mostram elevada e significativa correlação entre o $\mathrm{N}$ total do solo e o $\mathrm{N}$ mineralizado. PÖTTKER \& TEDESCO (1979) estudando a mineralização do $\mathrm{N}$ em diversos solos do RS observaram que a correlação entre o $\mathrm{N}$ total dos solos e o $\mathrm{N}$ mineralizado é maior do que as obtidas entre o $\mathrm{N}$ mineralizado e a MOS $(0,85$ e 0,75 , respectivamente) Desta forma, concluem que o teor de MOS e o teor de $\mathrm{N}$ total dos solos podem ser usados como índices da disponibilidade de $\mathrm{N}$, pois apresentam alta correlação com o $\mathrm{N}$ mineralizado anaerobiamente. Diversos autores argumentam que existe uma grande importância com relação ao conhecimento dos teores de MOS e de $\mathrm{N}$ total na predição do $\mathrm{N}$ potencialmente mineralizável, pois estes possuem elevada e significativa correlação entre si (PÖTTKER \& TEDESCO et al., 1979; INUBUSHI et al., 1985; CAMARGO et al., 1997; GONÇALVES et al., 2001; LI et al., 2003). O teor de argila do solo também é um importante atributo que deve ser considerado no estudo do $\mathrm{N}$ mineralizado, fato comprovado pela significativa associação entre o teor de argila do solo e o N absorvido pelas plantas. A correlação entre o teor de argila do solo e o $\mathrm{N}$ mineralizado foi relativamente alta $(\mathrm{r}=0,73 ; \mathrm{P}<0,01 ; \mathrm{n}=15)$. CAMARGO et al. (1997) estudando o potencial de mineralização do $\mathrm{N}$ aerobicamente observaram que o teor de argila influenciou na percentagem de mineralização do $\mathrm{N}$, o que provavelmente está atrelado a formação de complexos organo-minerais entre a argila e a MOS.

Ao se avaliar a relação $\mathrm{N}_{0}$ e o teor de $\mathrm{N}$ total do solo $\left(\mathrm{N}_{0} / \mathrm{Nt}\right)$, evidencia-se que os solos de Rosário do Sul e Paraíso do Sul apresentam a maior percentagem de mineralização, 14,8 e 15,5\% do N total, respectivamente (Tabela 2 ). Esta constatação pode estar atrelada ao fato de que o teor de $\mathrm{N}$ total destes solos é relativamente baixo, sendo os menores dentre os solos estudados, já o $\mathrm{N}$ mineralizado está entre os menores observados, quando comparado aos demais solos, além de que, o teor de C orgânico dos solos também é relativamente baixo. Este fato indica que a reserva orgânica de $\mathrm{N}$ destes solos é pequena, pois num curto espaço de tempo (24 semanas de incubação) houve elevada mineralização do $\mathrm{N}$, relativamente ao seu teor de $\mathrm{N}$ total, e, como o teor de argila destes solos é muito baixo, a proteção da MOS pela fração mineral é praticamente desprezível, restando à recalcitrância da MOS, a atividade microbiana e ao manejo do solo a regulação da decomposição da MOS. Estes fatos auxiliam a explicar os resultados encontrados. CAMARGO et al., 1997) comentam que a argila participa na formação de complexos organominerais insolúveis, o que pode resultar em proteção da MOS ao ataque microbiano, diminuindo a mineralização do $\mathrm{N}$.

Os solos de Dom Pedrito, Uruguaiana 1 e Camaquã apresentaram as menores percentagens para a relação $\mathrm{N}_{0} / \mathrm{Nt}$ (3,7, 4,7 e 4,9\%, respectivamente). Já os demais solos se situaram numa fixa intermediária, com uma média de $9,0 \%$ para a relação $\mathrm{N}_{0} / \mathrm{Nt}$. O solo de Dom Pedrito teve baixa relação $\mathrm{N}_{0} / \mathrm{Nt}$, fato que está associado ao elevado teor de $\mathrm{N}$ total deste solo e ao baixo teor de $\mathrm{N}$ mineralizado e, como o teor de $\mathrm{C}$ orgânico deste solo é relativamente baixo, evidenciase que a mineralização do $\mathrm{N}$ para este solo está atrelada ao teor de $\mathrm{C}$ orgânico do solo e a sua recalcitrância, e não ao teor de $\mathrm{N}$ total do solo. Os solos de Camaquã e Uruguaiana 2 apresentaram baixa percentagem $\mathrm{N}_{0} / \mathrm{Nt}$ devido à grande reserva orgânica de $\mathrm{N}$ destes solos, mesmo que tenham mineralizado considerável teor de $\mathrm{N}$, além de que, o teor de $\mathrm{C}$ orgânico destes solos é relativamente elevado, o que indica que a mineralização do $\mathrm{N}$ está atrelada ao teor de $\mathrm{C}$ orgânico, para alguns solos e, ao teor de $\mathrm{N}$ total para outros, e em alguns casos a ambos.

A correlação entre o $\mathrm{N}$ potencialmente mineralizável $\left(\mathrm{N}_{0}\right)$ e o $\mathrm{N}$ absorvido por plantas de arroz irrigado foi alta e significativa $(r=0,93 ; \mathrm{P}<0,01)$, demonstrando que os parâmetros $\mathrm{N}_{0}$ e $\mathrm{k}$ descritos pelo modelo exponencial simples proposto por STANFORD \& SMITH (1972) caracterizam adequadamente o N absorvido por plantas de arroz irrigado em função do $\mathrm{N}$ mineralizado, mesmo estimando valores de $\mathrm{N}_{0}$ ligeiramente superiores aos de $\mathrm{N}$ mineralizado. CAMARGO et al. (1997) também evidenciaram que o modelo exponencial simples proposto por STANFORD \& SMITH (1972) caracterizou adequadamente o N absorvido por plantas de milho, entretanto, os valores de $\mathrm{N}_{0}$ estimados pelo modelo foram ligeiramente inferiores aos de $\mathrm{N}$ mineralizado.

A constante da taxa de mineralização para os diferentes solos foi de 0,2078 semana $^{-1}$, na média, sendo superior às encontradas por STANFORD \& SMITH (1972) (0,054 semana $\left.{ }^{-1}\right)$, entretanto, inferiores às encontradas por CAMARGO et al. (1997) $(0,3196$ semana $^{-1}$ ). Segundo CAMARGO et al. (1997), a rápida mineralização inicial, seguida da estabilização por um período relativamente longo, pode, a princípio, explicar os valores elevados do k. LI et al. (2003) estudando o potencial de mineralização de seis solos chineses 
observou valores de $\mathrm{k}$ inferiores aos obtidos no presente trabalho, tanto em temperaturas de $25^{\circ}$ como de $35^{\circ} \mathrm{C}$. BENBI \& RICHTER (2002) comentam que os valores de $\mathrm{k}$ variam muito entre diferentes solos, e isso mostra que os solos não somente diferem com relação à fração de $\mathrm{N}$ orgânico ativo, mas também no "turnover" microbiano. A taxa de mineralização está significativamente associada ao teor de argila do solo ( $\mathrm{r}=0,77 ; \mathrm{P}<0,01 ; \mathrm{n}=15)$, assim como o verificado por INUBUSHI et al. (1985), que observaram que a taxa de mineralização está associada ao teor de argila do solo, bem como ao tipo de mineral de argila, o que é devido a capacidade de interação das frações argila e MOS.

No período inicial de incubação houve maior velocidade de mineralização, o que provavelmente afetou a taxa de mineralização. A ocorrência de intensa mineralização inicial pode ser estar associada à manipulação das amostras quando em pré-incubação. STANFORD \& SMITH (1972) recomendam desprezar as duas primeiras semanas de incubação a fim de iniciar os cálculos do potencial de mineralização e, CAMARGO et al. (1997) comentam que a ocorrência de taxas iniciais elevadas pode estar associada a um aumento da atividade microbiana devido ao manuseio das amostras. Nesta condição, a MOS é mais facilmente degradada, pois ocorre a destruição dos agregados e promove aeração do solo, estimulando diretamente ao aumento da atividade microbiana o que resultaria em elevada mineralização inicial do N. Entretanto, LI et al. (2003) argumentam que, em condição de campo, existem ciclos de umedecimento e secagem e este efeito pode ser assumido como semelhante ao feito de secagem ao ar e manipulação das amostras de solo quando em pré-incubação. Por isso, os autores comentam que não há razão para ignorar o $\mathrm{N}$ mineralizado inicialmente. Entretanto, quando se considera desde a primeira semana de incubação, a taxa de mineralização situa-se em média de 0,2078 semana $^{-1}$. Quando se considera a partir da segunda semana de incubação, a taxa diminui para 0,1572 semana $^{-1}$ e, para 0,1191 semana ${ }^{-1}$ quando se utilizam os dados de $\mathrm{N}$ mineralizado a partir da quarta semana de incubação.

A mineralização do $\mathrm{N}$ orgânico do solo está diretamente relacionada com o caráter lábil ou recalcitrante da MOS, o que afeta sobremaneira a velocidade de decomposição e mineralização do $\mathrm{N}$, além da atividade microbiana e das condições ambientais. CAMARGO et al. (1997) comenta que, mesmo não se estudando a atividade microbiana, pode-se, a partir do conhecimento da mineralização do $\mathrm{N}$ do solo, se estimar a potencial contribuição do N para com a nutrição mineral de plantas e, a partir deste conhecimento, gerar informações sobre a necessidade de adubação nitrogenada para as culturas, aperfeiçoando doses e épocas de aplicação do fertilizante nitrogenado.

\section{CONCLUSÕES}

A avaliação da mineralização do $\mathrm{N}$ permitiu separar os 15 solos em cinco grupos distintos quanto ao $\mathrm{N}$ mineralizado acumulado em 24 semanas. Houve maior velocidade de mineralização do $\mathrm{N}$ do período inicial de incubação até a quarta semana, diminuindo posteriormente.

O N mineralizado acumulado está altamente associado ao teor de $\mathrm{C}$ orgânico do solo, bem como o $\mathrm{N}$ absorvido pelas plantas ao teor de $\mathrm{N}$ mineralizado. Também existe elevada associação entre o teor de $\mathrm{N}$ absorvido por plantas de arroz irrigado e o $\mathrm{N}$ potencialmente mineralizável estimado pelo modelo de STANFORD \& SMITH (1972). O potencial de mineralização do $\mathrm{N}$ pode ser utilizado como índice da disponibilidade de $\mathrm{N}$ para a cultura do arroz irrigado.

A mineralização do $\mathrm{N}$ orgânico foi diferente entre os 15 solos, apresentando uma taxa de mineralização que variou entre 0,1492 a 0,2438 semana $^{-1}$

\section{REFERÊNCIAS}

BENBI, D.; RICHTER, J. A critical review of some approaches to modeling nitrogen mineralization. Biology and Fertility of Soils, v.35, p.168-183, 2002.

BLACK, C.A. Nitrogen: mineralization and immobilization. In: BLACK, C.A. (Ed). Soil-plant relationship. 2.ed. New York: John Wiley, 1968. p.419-452.

CAMARGO, F.A. et al. Nitrogênio orgânico do solo. In: CAMARGO, F.A.O.; SANTOS, F.A. (Eds). Fundamentos da matéria orgânica do solo: ecossistemas tropicais e subtropicais. Porto Alegre: Gênesis, 1999. p.117-137.

CAMARGO, F.A. et al. Potencial de mineralização do nitrogênio em solos do Rio Grande do Sul. Revista Brasileira de Ciência do Solo, v. 21, p.575-579, 1997.

COMISSÃO DE QUÍMICA E FERTILIDADE DO SOLO - RS/ SC. Manual de adubação e calagem para os estados do Rio Grande do Sul e Santa Catarina. Porto Alegre: SBCSNRS/EMBRAPA-CNPT, 2004. 400p.

EMBRAPA - CNPS. Manual de métodos de análise de solo. Brasília: EMBRAPA, 1977. 212p.

GONÇALVES, J.L.M. et al. Mineralização de nitrogênio em ecossistemas florestais naturais e implantados do Estado de São Paulo. Revista Brasileira de Ciência do Solo, v.25, p.601616, 2001.

INUBUSCHI, K. et al. Easily decomposable organic matter in paddy soil. VI. Kinetics of nitrogen mineralization in submerged soils. Soil Science and Plant Nutrition, v.4, p.563-572, 1985 .

LI, H. et al. Nitrogen mineralization in paddy soils of the Taihu Region of China under anaerobic conditions: dynamic and model fitting. Geoderma, v.115, p.161-175, 2003. 
NELSON, O.W.; SOMMERS, L.E. Total carbon, organic carbon, and organic matter. In: PAGE, A.L. et al. (Eds). Methods of soil analysis. Madison: ASA/SSSA, 1982. p.539-594.

NOVAIS, R.F.; SMYTH, T.J. Fósforo em solo e planta em condições tropicais. Viçosa: UFV, 1999. 339p.

PATRICK JR., W.H. Nitrogen transformations in submerged soils. In: STEVENSON, F.J. (Ed). Nitrogen in agricultural soils. Madison: ASA, CSSA, SSSA, 1982. Cap.12, p.449-466

PÖTTKER, D.; TEDESCO, M.J. Efeito do tipo e tempo de incubação sobre a mineralização da matéria orgânica e nitrogênio total em solos do Rio Grande do Sul. Revista Brasileira de Ciência do Solo, v.3, p.20-24, 1979.

RANNO, S.K. Estimativa da disponibilidade de fósforo para a cultura do arroz irrigado em solos do RS. 2004. 139f. Dissertação (Mestrado em Ciência do Solo) - Curso de Pós-graduação em Ciência do Solo, Universidade Federal de Santa Maria.
RHODEN, A.C. Potencial de mineralização anaeróbia do nitrogênio em solos de várzea do Rio Grande do Sul. 2005. 108f. Dissertação (Mestrado em Ciência do Solo) Curso de Pós-graduação em Ciência do Solo, Universidade Federal de Santa Maria.

STANFORD, G.; SMITH, S.J. Nitrogen mineralization potential of soils. Soil Science Society of American Proceedings, Madison, v.36, p.465-472, 1972.

STRECK, E. et al. Solos do Rio Grande do Sul. Porto Alegre: UFRGS/EMATER, 2002. 107p.

TEDESCO, M.J. et al. Análises de solo, plantas e outros materiais. 2.ed. Porto Alegre: Departamento de Solos Universidade Federal do Rio Grande do Sul, 1995. 174p. (Boletim técnico, 5).

VAHL, L.C. Fertilidade de solos de várzea. In: GOMES, A.S.; PAULETTO, E. (Eds). Manejo do solo e da água em áreas de várzea. Pelotas: Embrapa Clima Temperado, 1999. p.119162 . 